\title{
Hormone-Resistant Prostate Carcinoma
}

National Cancer Institute

\section{Source}

National Cancer Institute. Hormone-Resistant Prostate Carcinoma. NCI Thesaurus.

Code C114933.

Prostate carcinoma that does not respond to hormone therapy. 